PAPER

\title{
Traffic Distribution Scheme with ON-OFF Switching Based on Map Information in Heterogeneous Network
}

\author{
Takahiro Sekii ${ }^{1}$, Takaaki Onishi ${ }^{2}$ and Shigeaki Ogose ${ }^{3}$ \\ ${ }^{1,2,3}$ Graduate School of Engineering, Kagawa University \\ 2217-20 Hayashi-cho, Takamatsu, Kagawa 761-0396, Japan \\ E-mail: $\left\{{ }^{1}\right.$ s16g457, ${ }^{2}$ s16g453\}@stu.kagawa-u.ac.jp, ${ }^{3}$ ogose@eng.kagawa-u.ac.jp
}

\begin{abstract}
To process increasing data traffic, one of the most effective solutions is data offloading. Processing data traffic in heterogeneous networks (HetNets) based on loaded traffic conditions improves the user throughput. In this case, user traffic needs to be divided taking account of the difference in the transmission rate among networks. When a macro base station (BS) provides wireless resources for user equipment (UE) of good channel quality near the macro BS, higher user throughput is expected. However, at the macro cell edge, the transmission performance of UEs in a micro cell deteriorates. To reduce this inconvenience, by processing the loaded traffic appropriately using a macro BS and micro BS, we can obtain higher user throughput. This paper proposes wireless resource allocation based on loaded traffic conditions and the position of BSs with ON-OFF switching according to the channel quality in HetNets. Simulation results show the effectiveness of the proposed method, especially when the data loss ratio of the macro cell is low and that of micro cells is high. The proposed method is effective for a damage-resistant system against natural disasters and an automatic driving system in an intelligent transport system (ITS).
\end{abstract}

Keywords: wireless resource allocation, traffic distribution, heterogeneous network

\section{Introduction}

The amount of traffic in wireless networks is increasing rapidly. The requirements of $5 \mathrm{G}$ mobile networks are summarized into 1000 times the system capacity than that assuming long-term evolution (LTE), a high data rate of $10 \mathrm{Gbit} / \mathrm{s}$, delays of less than 1 ms, 100 times larger device connectivity than that assuming LTE, low cost and power saving[1]. Based on the internet of things (IoT), it is expected that the user density will be higher than that assuming LTE. It is assumed that the number of user equipments (UEs) on the internet will be 50 billion by the year 2020 and data traffic exceeding the system capacity will be generated[2]. Thus, the Institute of Electrical and Electronics Engineers (IEEE) has been considering the use of extremely high frequencies (EHF) for high-capacity communication[3]. However, EHF have problems of high path loss and propagation characteristics of strong straightness.

Heterogeneous networks (HetNets) consist of macro cells and small cells. A macro cell can cope with the movement of UEs. A small cell can limit the number of UEs with a small range of coverage. In the following scenarios, we found the usefulness of HetNets. Operation under the destruction of infrastructure caused by disasters such as earthquakes is necessary for safety and security. In this case, a wireless communication system with a function of autonomous operation without imposing a burden on the network is effective. In the field of intelligent transport systems (ITSs), on the other hand, a local dynamic map (LDM) for an automatic driving support system should be provided depending on the necessary speed of information delivery[4]-[6]. Static data such as physical road conditions do not need a high speed of transmission. Dynamic data such as traffic conditions and information on pedestrians on the road need real-time transmission.

The most effective method of having high user throughput under heavily loaded traffic conditions in HetNets is data offloading. In this method, loaded traffic is carried by both macro BSs and micro BSs. Regarding the offloading schemes, the following resource allocation methods have been proposed and studied: the round-robin method, the maximum carrier-to-interference power ratio (CIR) method and the proportional fair $(\mathrm{PF}) \operatorname{method}[7],[8]$.

Lightly loaded BSs exist according to the user distribution and traffic demand. Taking this fact into 
account, user throughput can be improved by processing traffic in multiple wireless networks according to the loaded traffic conditions[9]. A macro BS provides its wireless resources to UEs that exist within the highly loaded micro cells. As a result, the average user throughput can be improved. However, under heavily loaded traffic conditions, the wireless resource allocation of a macro cell may decrease user throughput.

In the world of IoT, a large number of devices are connected to a network. In this situation, the required quality of service (QoS) depends on the application. In this regard, existing resource allocation methods have not yet considered the requirements of the applications. It is obvious that we need to have a flexible packet flow that meets the QoS requirement. Thus, data offloading should be done while taking account of the types of information. The simplest method to guarantee the QoS required by applications is a socalled bandwidth -guarantee-service. Thus, in this paper, we deal with a bandwidth-guarantee-type roundrobin traffic offloading method.

The following is a problem of resource allocation in a HetNet to be solved. When we consider a HetNet operating with existing scheduling methods, macro cell resources are allocated to UEs that exist near the macro BS. However, at the macro cell edge, when the transmission performance of UEs in an overlaid micro cell deteriorates, it is difficult to meet the requirements.

Many cell load balancing and network selection schemes based on map information have been proposed[10]-[17]. Data offloading in HetNets is a hot and important topic. However, no sufficiently detailed studies on the above-mentioned circumstances have been carried out.

With the above-mentioned motivation, we propose a round-robin-based traffic offloading method that is simple but effective, especially in the case that the transmission quality of micro cells is low. Our proposed method can flexibly process the traffic by ONOFF switching of traffic offloading in accordance with the transmission quality and user distribution. The rest of this paper is organized as follows. Section 2 reviews the network selection scheme using map information and UE positions as well as conventional resource allocation schemes. In Sec. 3, we propose the resource allocation of a macro cell and ON-OFF switching for a traffic distribution scheme. In Sec. 4, computer simulation results are given to show the usefulness of the proposed scheme. Finally, Sec. 5 gives the conclusions of this paper.

\section{Related Works}

It is well known that one of the most effective methods of processing increasing data traffic is data offload- ing. Cell load balancing techniques include cell range expansion (CRE) and carrier aggregation (CA)[11][13], [17]. CRE is the technique in which a UE connects to a BS with the maximum value of the reference signal received power (RSRP) plus the CRE bias in the handover procedure.

If the carrier frequency of macro cells is different from that of micro cells, a UE tends to connect to both a macro BS and a micro BS based on the CA method. For effective data offloading, a network selection method supported by software design is needed[18]. A network selection method based on map information has been proposed to effectively select wireless networks[16]. A UE is a multiband terminal with a function of high-accuracy positioning such as through the use of quasi-zenith satellite system (QZSS).

The map information contains the expected throughput based on a channel quality map and a traffic map. The channel quality map consists of the average value of the received signal strength indicator (RSSI). The traffic map is needed under some loaded traffic conditions. For this purpose, a heterogeneous control server (HCS) is installed in a macro cell. A UE transmits the channel quality information of the BSs to construct the channel quality map. The HCS constructs the channel quality map to compute the average value of the RSSI for each micro cell BS in a macro cell. Also, each BS in a macro cell periodically transmits the information of the loaded traffic conditions to the HCS through the backbone network. Then, the HCS creates the map information and broadcasts it to UEs in a macro cell. On the basis of the map information, a UE selects a macro BS or a micro BS so as to obtain the highest expected throughput at the existing point of UE.

As we mentioned in Sec. 1, various resource allocation methods exist such as the round-robin, maximum CIR and PF methods[7], [8]. The characteristics of each method are described as follows. (i) Roundrobin method: This method has the highest user fairness. However, the frequency efficiency decreases easily. (ii) Maximum CIR method: This method focuses on improving the system user throughput and realizes higher frequency efficiency. However, the user fairness of this system is not so good. (iii) PF method: This method provides a good system user throughput while considering the fairness among users. This method is positioned between the round-robin and maximum CIR methods. In the PF method, the instantaneous throughput at the transmission time interval (TTI) is evaluated. At the present time slot, the resource is allocated to the user with the identification number of $k$ given by

$$
k=\operatorname{argmax} \frac{\left[r_{i}\right]^{\alpha}}{\left[\bar{R}_{i}\right]^{\beta}}(\text { for } 1 \leq i \leq N)
$$


Here, $N$ is the total number of users. $r_{i}$ is the instantaneous data rate at the present time slot for the $i$ th user and $\bar{R}_{i}$ is the average user throughput for the $i$ th user. $(\alpha=1, \beta=0)$ is used for the maximum CIR method, $(\alpha=1, \beta=1)$ is used for the PF method and ( $\alpha=0, \beta=1$ ) is used for the round-robin method. These methods have been used in appropriate areas taking into account their pros and cons. Network selection methods and resource allocation methods based on the idea of the PF method have been proposed[19]. In PF-based network selection, the predictive throughput is also calculated at the backbone sites. In our paper, the performance of the proposed method is compared with that of the conventional map information method. For this reason, we have indirectly made a comparison with the PF-based network selection method.

\section{Traffic Distribution Scheme with ON-OFF Switching}

\subsection{Traffic distribution scheme}

Figure 1 shows the system configuration of trafficdistributed communications. It is assumed that we have an HCS, the macro cell and micro cells for simplicity. A UE accesses two BSs, one is the macro cell and the other is a micro cell. Then, a UE distributes its loaded traffic. The transmission rate of the macro cell is generally smaller than that of a micro cell. If a UE carries out traffic-distributed communication without considering the difference in the transmission rate, efficient performance cannot be obtained.

It is assumed that the expected throughputs of the macro cell and a micro cell are $R_{a}$ and $R_{i}$, respectively. On the basis of these values, the loaded traffic, $S$, is divided into macro cell traffic and micro cell traffic as

$$
S=\underbrace{S \frac{R_{a}}{R_{a}+R_{i}}}_{\text {Macro cell }}+\underbrace{S\left(1-\frac{R_{a}}{R_{a}+R_{i}}\right)}_{\text {Micro cell }}
$$

where $R_{a}$ and $R_{i}$ are given by

$$
\begin{aligned}
R_{a} & =\frac{B_{a}}{N_{a}} \log _{2}\left(1+\gamma_{a}\right) \\
R_{i} & =\frac{B_{i}}{N_{i}} \log _{2}\left(1+\gamma_{i}\right)
\end{aligned}
$$

$B_{a}$ and $N_{a}$ denote the system bandwidth and the number of active UEs in the macro cell, respectively. In the same way, $B_{i}$ and $N_{i}$ denote the system bandwidth and the number of active UEs in a micro cell, respectively. $\gamma_{a}$ and $\gamma_{i}$ denote the signal-to-noise power ratio (SNR) in the macro cell and a micro cell, respectively.

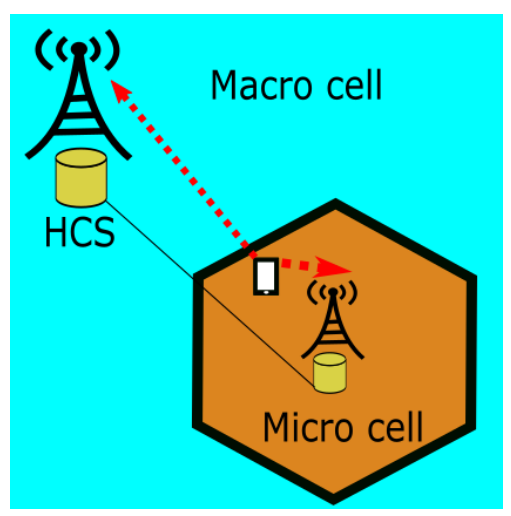

Fig. 1 System configuration of traffic-distributed communications

Note that the throughput should be calculated after resource block scheduling in accordance with the user traffic, which is generated independently and randomly. However, in our paper, cases of text data, such as Web traffic which is generated one after another, are not considered for simplicity.

In an LDM, frequency fairness is more important than system throughput fairness. This is because the frequency resource for dynamic data transmission is not always preferentially assigned. Thus, the resource allocation in our paper deals with a round-robin-based system.

A UE obtains the value of SNR corresponding to the macro cell and each micro cell from the pilot signal. The macro BS obtains the information on the number of active UEs within micro cells through the backbone network in order to calculate the expected throughput. Consideration of the instantaneous interference signal power is not easy. Thus, SNR is used instead of the signal-to-interference plus noise power ratio (SINR).

\subsection{Resource allocation of macro cell}

The macro BS transfers its wireless resources to one of the overlaid highly loaded micro BSs. As a result, a UE consumes wireless resource $B_{n}$ as follows:

$$
B_{n}= \begin{cases}\frac{B_{i}}{N_{i}}+\frac{B_{a}}{N_{a}} & \left(n=n_{\max }\right) \\ \frac{B_{i}}{N_{i}} & \text { (otherwise) }\end{cases}
$$

where $n(n=1,2, \cdots, N)$ denotes the identification number of each micro cell. Here, $N$ is the total number of micro BSs that are overlaid with a specified macro BS. $n_{\max }$ denotes the identification number of the micro cell that is allocated wireless resources by the macro BS. Under heavily loaded traffic conditions, the advantageousness of the traffic distribution scheme decreases when a UE is located far from the macro BS. This is because of the lower signal transmission quality. To cope with this inconvenience, a weighted traf- 


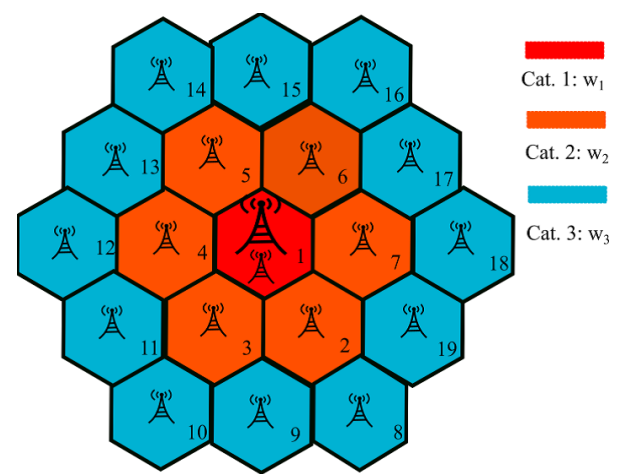

Fig. 2 Micro cell categories and the weighting coefficients

fic distribution is introduced. In this method, the BS provides wireless resources according to the weighting coefficient based on the position of the BSs.

When we assume the layout of 19 hexagonal cells as shown in Fig.2, micro cells are divided into three categories according to the distance from the macro cell BS. A micro cell of Category 1 exists at the center of the macro cell. Values of the weighting coefficient for each category are also given in the same figure. The weighting coefficient corresponds to the importance in the traffic distribution. The weighting coefficients for Category 1, Category 2 and Category 3 are $w_{1}, w_{2}$ and $w_{3}$, respectively. The evaluation function for the micro BSs with identification number $n$ depending on the loaded traffic conditions is defined by $F_{n}$, where

$$
F_{n}=\left\{\begin{array}{lll}
N_{i} \times w_{1} & (n=1) & \text { Cat. } 1 \\
N_{i} \times w_{2} & (n=2, \cdots, 7) & \text { Cat. } 2 \\
N_{i} \times w_{3} & (n=8, \cdots, 19) & \text { Cat. } 3
\end{array}\right.
$$

In this case, $n_{\max }$ in Eq. (7) is given by

$$
n_{\max }=\operatorname{argmax}\left(F_{n}\right)
$$

when we take into account the weighting coefficient.

\subsection{ON-OFF switching}

When micro cell $n$ is not in Category 1 , the macro BS operates in the traffic distribution scheme with ON-OFF switching as follows:

$\begin{cases}\mathrm{ON} & \left(\sum_{j=1}^{J} \gamma_{a, j}-\gamma_{i_{n}, j} \leq \epsilon\right) \\ \mathrm{OFF} & \left(\sum_{j=1}^{J} \gamma_{a, j}-\gamma_{i_{n}, j}>\epsilon \text { or } n=n_{\max }\right)\end{cases}$

Here, $j(j=1,2, \cdots, J)$ are the identification numbers of the UEs associated with the macro BS. $\gamma_{a, j}$ is the SNR for user $j$ in the macro cell. $\gamma_{i_{n}, j}$ is the SNR for user $j$ in micro cell $n$. $\epsilon$ is the threshold value of the difference in SNR between the macro BS and micro BS $n$.

\section{Computer Simulation}

To show the effectiveness of resource allocation based on the traffic distribution scheme with ON-OFF switching, we conducted computer simulations. $\lambda$ is the loaded traffic condition index, which is defined by the average number of active users per second in a micro cell[20]. $\lambda$ follows a Poisson distribution. It is assumed that UEs are uniformly distributed in the macro cell area. It is also assumed that data communications between the macro BS and a micro BS are realized through a wired line. Thus, a UE knows the loaded traffic conditions before starting transmission to obtain the announcement through the broadcast channel. For a file size of $S$, the user throughput of the proposed scheme, $U$, is calculated as

$$
\begin{array}{r}
U=\left\{\begin{aligned}
\frac{S}{t_{a}} & \left(t_{a} \geq t_{i}\right) \\
\frac{S}{t_{i}} & \text { (otherwise) }
\end{aligned}\right. \\
t_{a}=\frac{S \frac{R_{a}}{R_{a}+R_{i}}}{R_{a}} \\
t_{i}=\frac{S\left(1-\frac{R_{a}}{R_{a}+R_{i}}\right)}{R_{i}}
\end{array}
$$

where the processing times of the macro cell and a micro cell are $t_{a}$ and $t_{i}$, respectively. In the conventional scheme, user throughput is calculated by Eqs. (3) and (4). The computer simulation parameters are listed in Table 1. The propagation path loss models were urban macro cell (UMa) and urban micro cell (UMi) models with non-line-of-sight (NLoS) deployed by ITU-R[21]. The transmitter power control is not considered for simplicity. Map information consists of the signal transmission quality, loaded traffic, and so forth. In this method, network selection is conducted according to the predicted throughput of each BS calculated from the map information.

\subsection{Weighting pattern}

By combining the weighting coefficients $w_{1}, w_{2}$ and $w_{3}$, we have 13 different weighting patterns as shown in Table 2. For these weighting patterns, the average user throughput was obtained by computer simulation. The value of $\lambda$ for the micro cells is tentatively set as 0.5 to examine the effect of the weighting operation.

Figure 3 shows the average user throughput based on the values of the weighting coefficients. In a conventional scheme, the average user throughput does not change with the weighting pattern. When $w_{1}$ is large, we have higher user throughput. This is because the macro BS allocates wireless resources for UEs with high channel quality. For the weighting pattern 13 of 
Table 1 Computer simulation parameters

\begin{tabular}{llc}
\hline & Macro cell & Micro cell \\
\hline Cell layout & \multicolumn{2}{c}{19 hexagonal cells } \\
\hline Cell radius $[\mathrm{m}]$ & 216 & 50 \\
\hline Carrier frequency $[\mathrm{MHz}]$ & 2,000 & 2,400 \\
\hline System bandwidth $[\mathrm{MHz}]$ & 10 & 10 \\
\hline Transmission power $[\mathrm{dBm}]$ & 30 & 23 \\
\hline $\begin{array}{l}\text { Noise power spectrum den- } \\
\text { sity }[\mathrm{dBm} / \mathrm{Hz}]\end{array}$ & -174 \\
\hline Number of base stations & 1 & 19 \\
\hline Path loss model & UMa & UMi \\
& (NLoS) & $(\mathrm{NLoS})$ \\
\hline Number of UE & \multicolumn{2}{c}{100} \\
\hline $\begin{array}{l}\text { Base station antenna height } \\
\text { [m] }\end{array}$ & 25 & 10 \\
\hline UE antenna height $[\mathrm{m}]$ & \multicolumn{2}{c}{1.5} \\
\hline Channel model & \multicolumn{2}{c}{ Rayleigh fading } \\
\hline File size [Mbyte] & \multicolumn{2}{c}{$1,2,4$} \\
\hline Weighting coefficient & $0.5,2.5$ \\
\hline Loaded traffic index & \multicolumn{2}{c}{}
\end{tabular}

Table 2 Weighting patterns

\begin{tabular}{cccc}
\hline Weighting pattern & $w_{1}$ & $w_{2}$ & $w_{3}$ \\
\hline 1 & 1 & 1 & 1 \\
\hline 2 & 1 & 1 & 2 \\
\hline 3 & 1 & 2 & 1 \\
\hline 4 & 1 & 2 & 2 \\
\hline 5 & 1 & 2 & 4 \\
\hline 6 & 1 & 4 & 2 \\
\hline 7 & 2 & 1 & 1 \\
\hline 8 & 2 & 1 & 2 \\
\hline 9 & 2 & 1 & 4 \\
\hline 10 & 2 & 2 & 1 \\
\hline 11 & 2 & 4 & 1 \\
\hline 12 & 4 & 1 & 2 \\
\hline 13 & 4 & 2 & 1 \\
\hline & & &
\end{tabular}

$w=\left(w_{1}, w_{2}, w_{3}\right)=(4,2,1)$, the largest improvement of the average user throughput of $8 \%$ is obtained compared with the conventional method. For weighting patterns $2,5,9$, no improvement of the user throughput is obtained. Here, the conventional scheme is the previously mentioned network selection method.

\subsection{ON-OFF switching operation}

We now look at the simulation results for user throughput versus $\epsilon$ with and without ON-OFF switching when $w=(4,2,1)$.

Figure 4 shows the average user throughput under lightly loaded traffic conditions. The user throughput of the conventional method is also illustrated for comparison. The improvement of the average user throughput without ON-OFF switching is larger than that with ON-OFF switching. Under lightly loaded traffic conditions, a macro BS has sufficient bandwidth

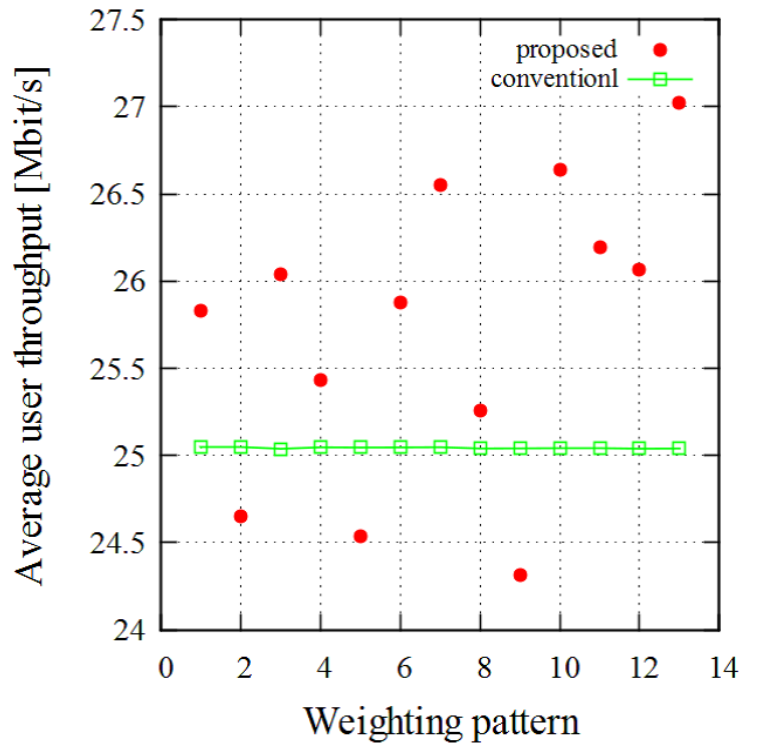

Fig. 3 Effect of weighting on average user throughput

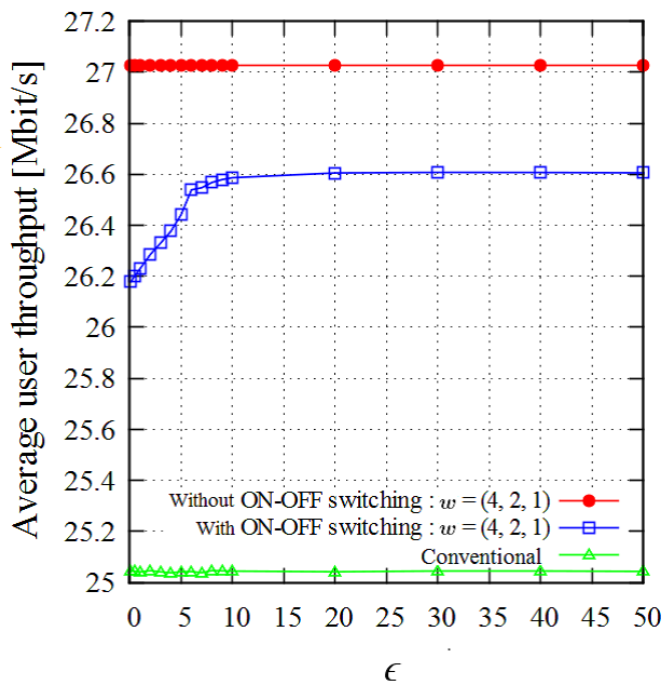

Fig. 4 Average user throughput $(\lambda=0.5)$

to process the traffic.

Figure 5 shows the average user throughput under heavily loaded traffic conditions. The macro BS does not have sufficient bandwidth to process the traffic. As a result, the average user throughput with ON-OFF switching is higher than that without ONOFF switching. When $\epsilon$ is 11, a maximum throughput of $15.4 \mathrm{Mbit} / \mathrm{s}$ is obtained with ON-OFF switching. However, the average user throughput with ON-OFF switching decreases as $\epsilon$ increases. This is because, ON-OFF switching behaves similarly to in the conventional method.

4.3 Average user throughput based on loaded traffic conditions

Under lightly loaded traffic conditions, it is assumed that the number of highly loaded micro BSs 


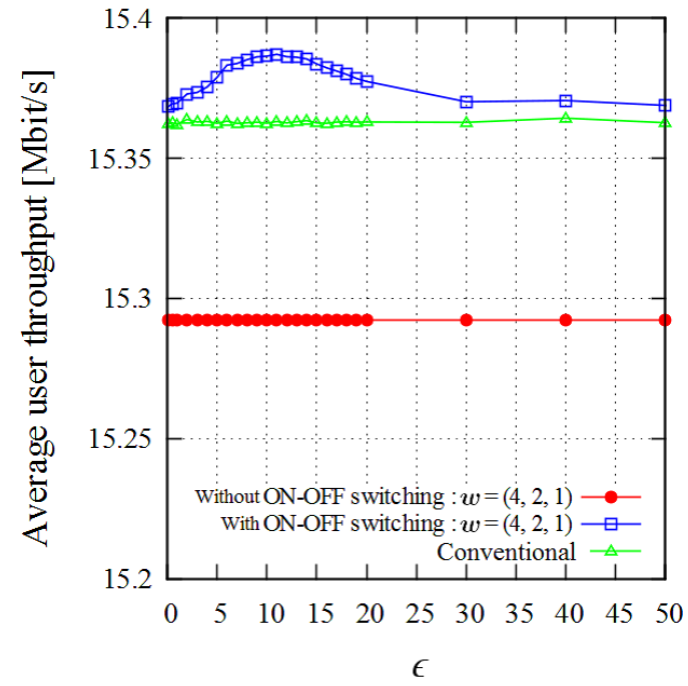

Fig. 5 Average user throughput $(\lambda=2.5)$

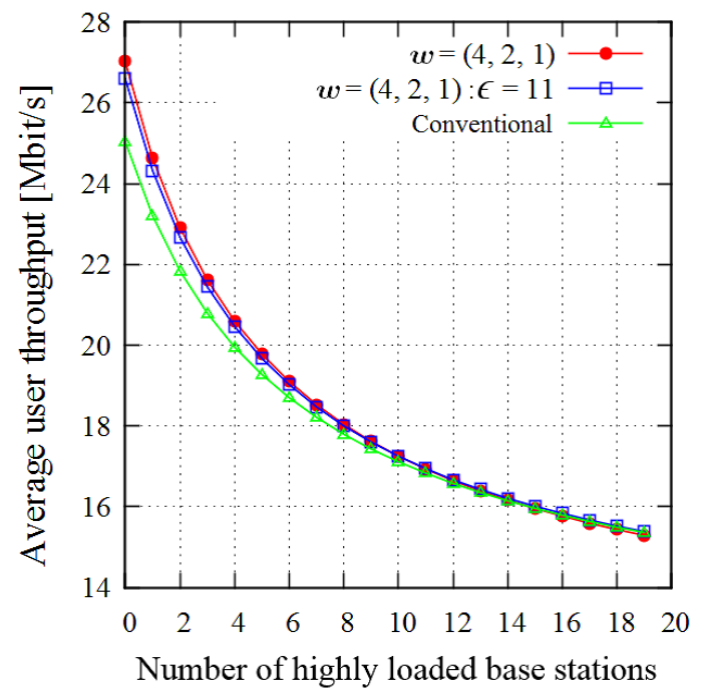

Fig. 6 Average user throughput vs number of highly loaded BSs

is 0. Conversely, under heavily loaded traffic conditions, it is assumed that the number of highly loaded micro BSs is 19 . Here, we set the value of $\lambda$ for highly loaded micro BSs as 2.5.

Figure 6 shows the average user throughput versus the number of highly loaded micro BSs. The red line represents the case without ON-OFF switching and the blue line represents the case with ON-OFF switching. The value of $\epsilon$ for ON-OFF switching is set to 11 . Highly loaded BSs are selected randomly. For ON-OFF switching, the average user throughput is improved by 0.15-6.2\% compared with the conventional method. On the other hand, without ON-OFF switching the average user throughput is improved by up to $7.9 \%$.

Figures 7 and 8 show cumulative frequencies of user throughput. When the number of highly loaded BSs is 0 and 19, respectively. As shown in Fig.7, when the cumulative frequency is 0.8 , the average user throughput is around $40 \mathrm{Mbit} / \mathrm{s}$. On the other hand, for the

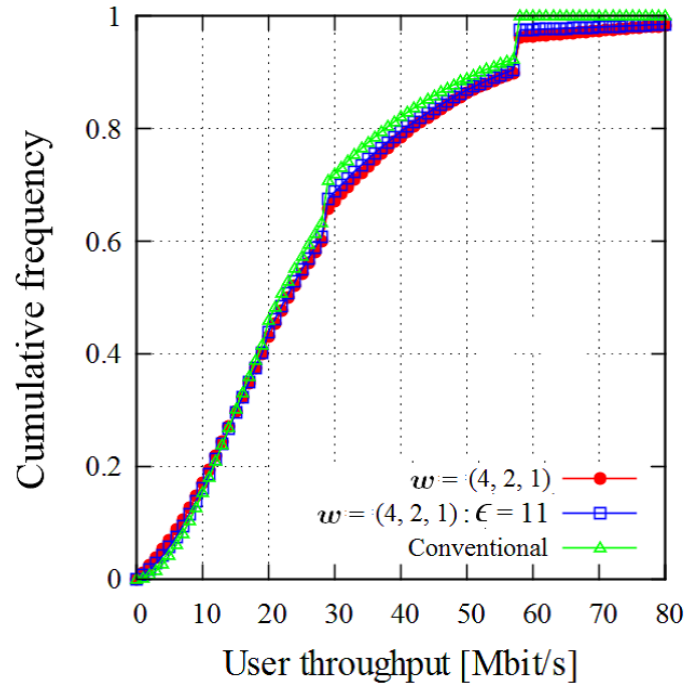

Fig. 7 Cumulative frequency of average user throughput under lightly loaded traffic conditions

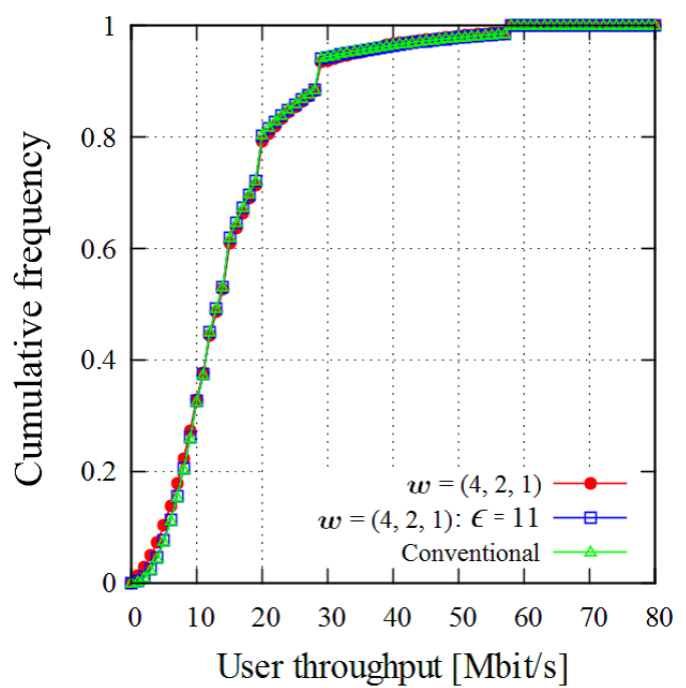

Fig. 8 Cumulative frequency of average user throughput under heavily loaded traffic conditions

same cumulative frequency in Fig.8, the average user throughput is about $20 \mathrm{Mbit} / \mathrm{s}$ because of the heavy traffic. The cumulative frequency curve of the user throughput increases stepwisely. This is because of the existence of three micro cell categories with different weighting coefficients.

\subsection{Dependence of user throughput on data loss ratio}

When we take account of the data loss at BSs, the user throughput, $U$, is calculated as follows:

$$
U= \begin{cases}\frac{S\left(1-L_{a}\right)}{t_{a}} & \left(t_{a} \geq t_{i}\right) \\ \frac{S\left(1-L_{i}\right)}{t_{i}} & \text { (otherwise) }\end{cases}
$$

where $L_{a}$ and $L_{i}$ are the data loss ratios of the macro cell and the micro cells, respectively. 


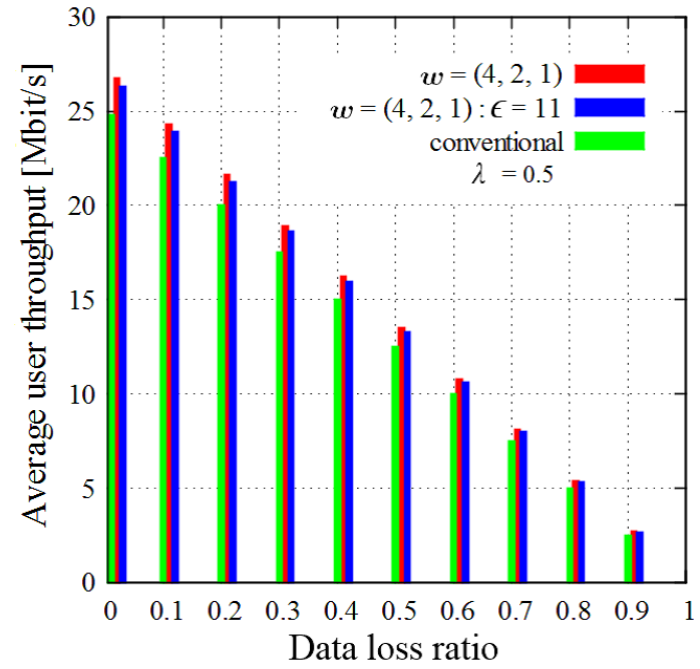

Fig. 9 Average user throughput vs data loss ratio $\left(L_{a}=L_{i}\right)$

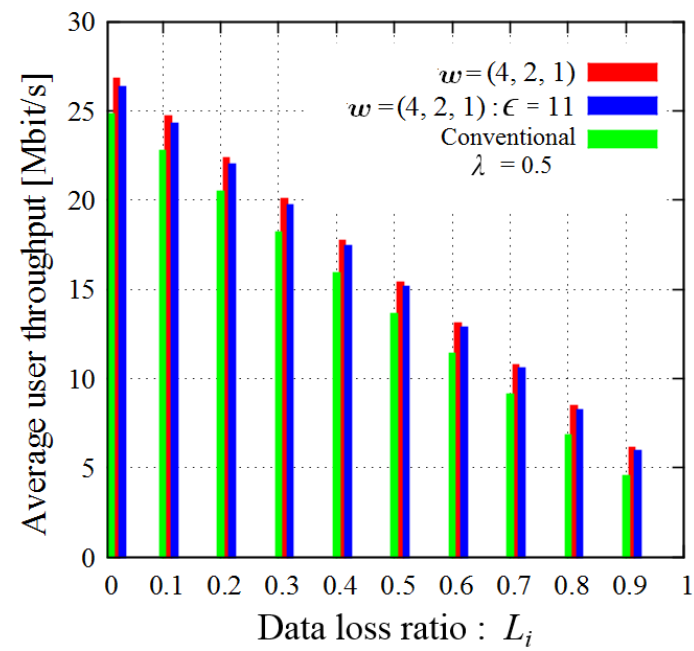

Fig. 10 Average user throughput vs $L_{i}\left(L_{a}=10^{-6}\right)$

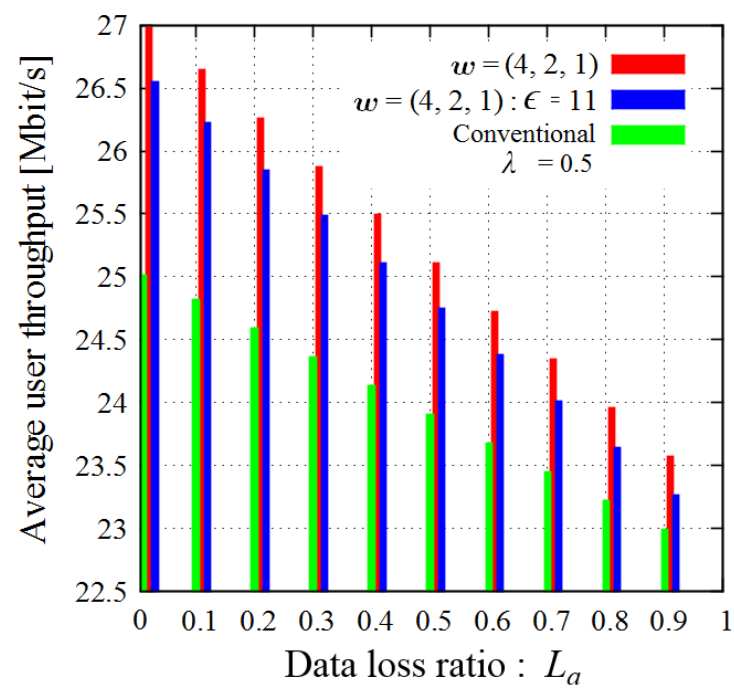

Fig. 11 Average user throughput vs $L_{a}\left(L_{i}=10^{-6}\right)$
Figure 9 shows the average user throughput in the case of $L_{a}=L_{i}$. We assume a lightly loaded traffic density simply to determine the effect of data loss. As the data loss ratio increases, the average user throughput decreases. Because the data loss ratio for the macro cell and micro cell is identical, we have similar performances both with and without the ON-OFF method. It is shown that some improvement is obtained by the proposed method compared with the conventional method.

Figures 10 and 11 show the average user throughput under lightly loaded traffic conditions. $L_{a}$ or $L_{i}$ is fixed as $10^{-6}[22]$. The transmission data for the macro cell is generally more reliable than that for a micro cell. As $L_{i}$ decreases, the improvement ratio decreases. In Fig.10, it is shown that when $L_{i}=0.9$, the maximum improvement of the average user throughput is obtained. The improvement ratio both with and without ON-OFF switching is about $44 \%$ compared with the conventional method. When $L_{a}$ is between 0 and 0.9 , the maximum improvements of the average user throughput with and without ON-OFF switching are $1.1 \%$ and $2.3 \%$, respectively.

Figures 12 and 13 show the average user throughput under the heavily loaded traffic condition of $\lambda$ $=2.5$. Similar performance is obtained for the proposed scheme and the conventional scheme. As shown in Fig.12, when $L_{i}=0.9$, the maximum improvements of the average user throughput with and without $\mathrm{ON}-\mathrm{OFF}$ switching are $1.7 \%$ and $6.0 \%$, respectively. Similarly, from Fig.13, we found that as $L_{a}$ increases, the throughput improvement ratio increases. A slightly higher improvement ratio is obtained without ON-OFF switching method than with the ONOFF switching method. As we mentioned in Sec. 1, we have higher data loss when the BS is out of order due to a disaster such as an earthquake. Also in the LDM in an ITS, providing dynamic data through micro cells may cause higher data loss[23], [24]. Thus, in these situations, the effectiveness of our proposed method is expected. When we compare the average user throughput of the proposed method and the conventional network selection method with map information, the proposed method can provide higher throughput.

Resource selection is difficult at the macro cell edge in cases of the PF method. Thus, from the viewpoint of frequency resource fairness, our proposed method is superior to the PF method. A UE should send information on the signal transmission quality to the HCS via the BS from time to time. Signal transmission quality does not fluctuate with time as long as a UE travels with a low speed. Note that a micro BS generally supports low-mobility communication. In this case, the frequency of reports on signal transmission quality is expected to be low. Collected data on SNR or SINR can be sent not separately but together dur- 


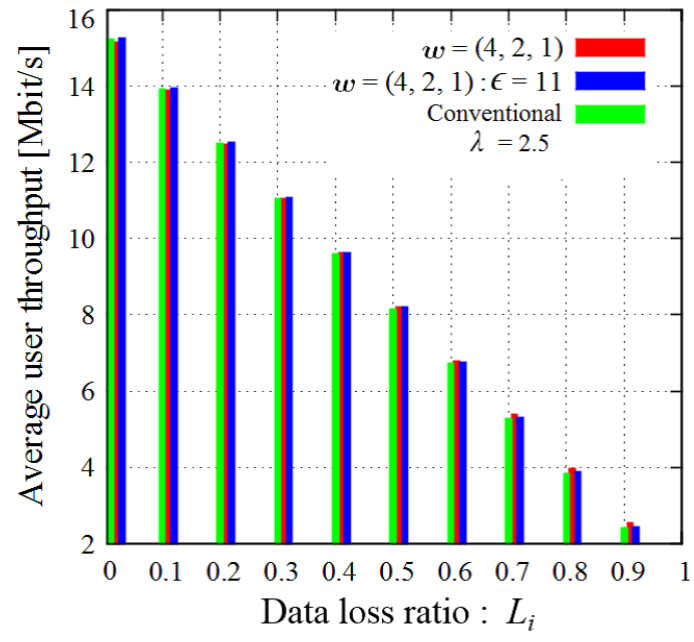

Fig. 12 Average user throughput vs $L_{i}\left(L_{a}=10^{-6}\right)$

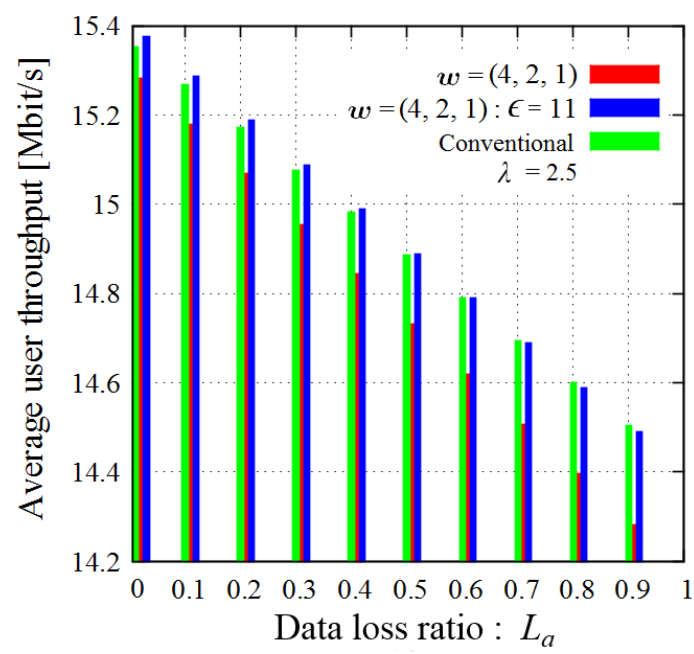

Fig. 13 Average user throughput vs $L_{a}\left(L_{i}=10^{-6}\right)$

ing the idling period when important communication is not in progress. Furthermore, when the map information is stored in a cloud server as an LDM in an ITS, we can solve the map information update and sharing problems. By doing so, we can prevent the network from traffic congestion due to the exchange of map information.

\section{Conclusions}

This paper proposed a wireless resource allocation method for traffic offloading in HetNets. The proposed method is characterized by traffic distribution based on loaded traffic conditions at each BS, weighting by the positions of BSs, and ON-OFF switching according to the transmission channel quality. Computer simulation results show that an improvement of the average user throughput of $0.15-7.9 \%$ is obtained. The improvement ratio of the average user throughput with and without ON-OFF switching is $44 \%$ when the data loss rates of the macro cell and each micro cell are $10^{-6}$ and 0.9 , respectively. The proposed method is effective in cases of system damage due to a disaster and with an LDM-based automatic driving system in an ITS. Future works include consideration of the time variation of loaded traffic, packet loss due to queuing delays, and the real user distribution based on user movement. Introducing a genetic algorithm (GA) and artificial intelligence (AI) will be effective for traffic prediction and resource scheduling. Also, it may reduce the data exchange/calculation of map information. Further study is needed on this matter.

\section{References}

[1] DOCOMO White Paper: http://www.nttdocomo.co.jp/ english/corporate/technology/whitepaper_5g/. (Accessed 26 Dec. 2016)

[2] D. Evans: The Internet of Things: How the next evolution of the Internet is changing everything, CISCO White Paper, 2011.

[3] A. Maltsev et al.: Channel models for IEEE 802.11ay, IEEE 802.11-15/1150r6, 2016.

[4] Intelligent Transport System (ITS) Vehicular Communications Basic Set of Applications Local Dynamic Map (LDM), ETSI EN 302895 (V1.1.1), 2014.

[5] H. Shimada, A. Yamaguchi, H. Takada and K. Sato: Implementation and evaluation of local dynamic map in safety driving-systems, J. Transport. Technol., Vol.5, No.2, pp.102-112, 2015.

[6] S. Kamijo, Y. Gu and L. Hsu: Autonomous vehicle technologies: Localization and mapping, IEICE Fundam. Rev., Vol.9, No.2, pp.131-141, 2015.

[7] H. Kim and Y. Han: A proportional fair scheduling for multicarrier transmission systems, IEEE Commun. Lett., Vol.9, No.3, pp.384-386, 2005.

[8] B. Yaser and K. Ivica: Performance evaluation of proportional fairness scheduling in LTE, Proc. WCECS 2013, pp.712-717, 2013.

[9] H. Kim, G. Veciana, X. Yang and M. Venkatachalam: Distributed $\alpha$-optimal user association and cell load balancing in wireless networks, IEEE/ACM Trans. Networking, Vol.20, No.1, pp.177-190, 2011.

[10] L. Wang, C. Wang and F. Adachi: Load-balancing spectrum decision for cognitive radio networks, IEEE J. Sel. Areas Commun., Vol.29, No.4, pp.757-769, 2011.

[11] T. Kudo and T. Ohtsuki: Cell range expansion using distributed Q-learning in heterogeneous networks, EURASIP J. Wireless Commun. and Networking, Article 61, pp.1-10, 2013.

[12] S. Sun, W. Liao and W. Chen: Traffic offloading with rate-based cell range expansion offsets in heterogeneous networks, Proc. 2014 IEEE WCNC, pp.2833-2838, 2014.

[13] F. Bénézit, S. Elayoubi, R. Indre and A. Simonian: Modelling load balancing and carrier aggregation in mobile networks, Proc. WiOpt 2014, pp.78-85, 2014.

[14] Y. Chen, J. Li, H. Chen, Z. Lin, G. Mao and J. Cai: A belief propagation approach for distributed user associa- 
tion in heterogeneous networks, Proc. IEEE PIMRC'14, pp.1793-1797, 2014.

[15] A. Gupta, H. Dhillon, S. Vishwanath and J. Andrews: Downlink multi-antenna heterogeneous cellular network with load balancing, IEEE Trans. Commun., Vol.62, No.11, pp.4052-4067, 2014.

[16] J. Kuboniwa, Y. Miyake, S. Kameda, A. Taira, H. Oguma, N. Suematsu, T. Takagi and K. Tsubouchi: High efficient network selection scheme using location information for heterogeneous wireless system, Proc. IEEE WCNC 2015, pp.391-396, 2015.

[17] H. Wang, C. Rosa and K. Pedersen: Dual connectivity for LTE-advanced heterogeneous networks, J. Wireless Networks, Vol.22, No.4, pp.1315-1328, 2016.

[18] S. Sun, K. Adachi, P. H. Tan, Y. Zhou, E. Kurniwan and J. Joung: Cognitive HetNet for 5G, Proc. IEEE VTS APWCS 2016, pp.37-38, 2016.

[19] T. Fukunaga, Y. Yuda, M. Hoshino and K. Higuchi: Decentralized user association for $(p, \alpha)$-proportional fairbased system throughput maximization in cellular networks, Proc. ISWCS 2015, pp.25-28, 2015.

[20] 3GPP TR36.814 (V9.0.0): Further advancements for EUTRA physical layer aspects, 2010.

[21] Guidelines for evaluation of radio interface technologies for IMT-Advanced, ITU-R Report M.2135, 2008.

[22] V. Tikhvinskiy, G. Bochechka and A. Gryazev: QoS requirements as factor of trust to $5 \mathrm{G}$ network, J. Telecommun. and Inf. Technol., Vol.14, No.1, pp.3-8, 2016.

[23] R. Kitamura, R. Shinkuma and T. Takahashi: Distributed scheduling for QoS control in uplink CDMA, Proc. 2005 APCC, pp.97-101, 2005.

[24] S. Niida, T. Inoue and Y. Takeuchi: Fundamental analysis of two-layered scheduling algorithm for a wireless packet system, Proc. IEEE VTC 2006, pp.446-450 2006.

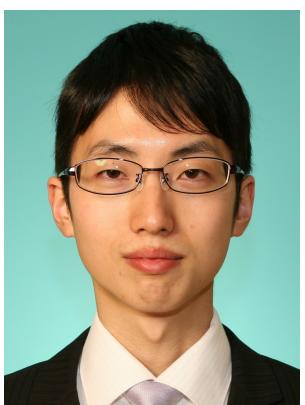

Takahiro Sekii received his B.S. degree in electronics and information engineering from $\mathrm{Ka}$ gawa University, Japan, in 2016. His research interests are in the area of heterogeneous wireless networks. He is a member of IEEE.

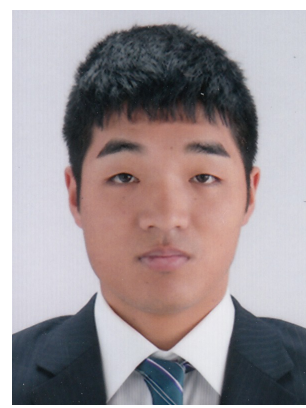

Takaaki Onishi received his B.S. degree in electronics and information engineering from Kagawa University, Japan, in 2016. His research interests include wireless sensor networks and energy harvesting. He is a member of IEEE.

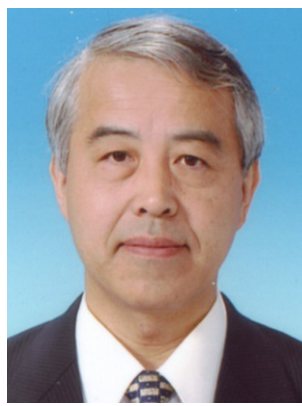

Shigeaki Ogose received his B.S. and M.S. degrees in electrical and electronics engineering from Hiroshima University in 1977 and 1979, respectively. He received his Ph.D. degree from Kyoto University in 1988. In 1977, he joined the Electrical Communication Laboratory (ECL), Nippon Telegraph and Telephone Corporation (NTT). Since 1998, he has been with Kagawa University. He is currently a Professor of the Department of Electronics and Information Engineering, Faculty of Engineering. His research interests include wireless communications and bio-sensor networks. He has co-authored several books on wireless communications such as Wireless Communications in the 21st Century. He is a member of IEEE, IEICEJ, IEEJ, ICME, and RISP.

(Received February 20, 2017: revised June 15, 2017) 